\title{
Regulation of Diurnal Variation of Cholesterol $7 \alpha$-hydroxylase (CYP7A1) Activity in Healthy Subjects
}

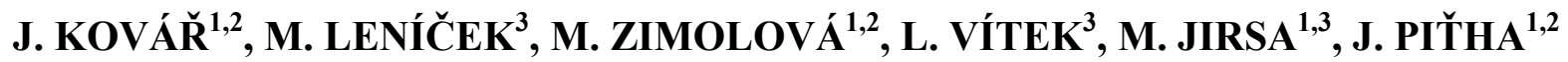 \\ ${ }^{1}$ Institute for Clinical and Experimental Medicine, Prague, Czech Republic, ${ }^{2}$ Centre of \\ Cardiovascular Research, Prague, Czech Republic, ${ }^{3}$ Institute of Clinical Biochemistry and \\ Laboratory Diagnostics, First Faculty of Medicine, Charles University in Prague, Czech Republic
}

Received January 26, 2009

Accepted March 26, 2009

On-line June 19, 2009

\section{Summary}

Cholesterol $7 \alpha$-hydroxylase (CYP7A1), the key regulatory enzyme of bile acid synthesis, displays a pronounced diurnal variation. To better understand the regulation of CYP7A1 activity, three daylong examinations were carried out in 12 healthy men. The concentrations of $7 \alpha$-hydroxycholest-4-en-3-one (C4), a surrogate marker of CYP7A1 activity, bile acids (BA), insulin, glucose, nonesterified fatty acids, triglycerides, and cholesterol were measured in serum in 90-min intervals from 7 AM till 10 PM. To lower and to increase BA concentration during the study, the subjects received cholestyramine and chenodeoxycholic acid (CDCA), respectively, in two examinations. No drug was used in the control examination. There was a pronounced diurnal variation of $\mathrm{C} 4$ concentration with a peak around $1 \mathrm{PM}$ in most of the subjects. The area under the curve (AUC) of C4 concentration was five times higher and three times lower when subjects were treated with cholestyramine and $C D C A$, respectively. No relationship was found between AUC of $\mathrm{C} 4$ and AUC of BA concentration, but AUC of C4 correlated positively with that of insulin. Moreover, short-term treatment with cholestyramine resulted in about $10 \%$ suppression of glycemia throughout the day. Our results suggest that insulin is involved in the regulation of diurnal variation of CYP7A1 activity in humans.

\section{Key words}

Cholesterol $7 \alpha$-hydroxylase • Bile acid • Insulin • Glucose • Cholestyramine

\section{Corresponding author}

J. Kovár, Institute for Clinical and Experimental Medicine, Laboratory for Atherosclerosis Research, Vídeňská 1958/9, 14021 Prague 4, Czech Republic. Fax: +420 241721666. E-mail: jan.kovar@medicon.cz

\section{Introduction}

Cholesterol $7 \alpha$-hydroxylase (CYP7A1) catalyzes the first key regulatory step in the synthesis of bile acids (BA) from cholesterol (Chiang et al. 1998, Gilardi et al. 2007). The synthesized bile acids are conjugated, exported into bile, stored in the gallbladder and, depending on metabolic needs, secreted into the intestine to facilitate fat absorption. Approximately $95 \%$ is then reabsorbed and returned to the liver. BA returning from the enterohepatic cycle downregulate CYP7A1 activity and they exert such an effect at the level of gene transcription (Ramirez et al. 1994). BA bind to nuclear receptor - farnesoid X receptor (FXR) and turn on the expression of small heterodimer partner (SHP) which then blocks the CYP7A1 gene transcription. Alternatively, they can downregulate CYP7A1 by nonFXR-dependent pathway (Gilardi et al. 2007). Moreover, BA absorbed in the intestine activate synthesis of fibroblast growth factor 19 (FGF19), that signals to the liver through its receptor FGFR4 and synergistically downregulates CYP7A1 gene expression (Inagaki et al. 2005).

CYP7A1 activity varies during the day - the circadian variation of the enzyme activity is well documented in rodents (Noshiro et al. 1990) and displays a pronounced circadian variation in humans (Gälman et al. 2005). The peak of the enzyme activity in humans is observed shortly after midday, which is rather difficult to reconcile with the fact that at this time the inhibition by BA returning from the enterohepatic cycle should prevail. Interestingly, it was recently demonstrated that 
BA seem to directly affect the glucose metabolism through both FXR-independent and FXR-dependent pathways (De Fabiani et al. 2003, Ma et al. 2006).

Therefore, to better understand the mechanisms behind the regulation of CYP7A1 activity, we measured the diurnal variation of $7 \alpha$-hydroxycholest-4-en-3-one (C4) concentration, a surrogate marker of CYP7A1 activity in vivo (Axelson et al. 1988), and changes of BA, insulin, glucose, nonesterified fatty acids (NEFA), triglycerides, and cholesterol concentration in healthy volunteers. The study was carried out under the conditions when BA synthesis was markedly increased or suppressed by short-term treatment with bile acid sequestrant cholestyramine or chenodeoxycholic acid (CDCA), respectively.

\section{Methods}

\section{Subjects and study design}

Twelve male volunteers (age: $32.1 \pm 4.0$ years, BMI: $25.7 \pm 4.3 \mathrm{~kg} / \mathrm{m}^{2}$ ) were included into the study that consisted of three day-long examinations. One of these examinations served as a control examination (without any drug), while the other two examinations studied the effect of short-term administration of cholestyramine (Questran ${ }^{\circledR}$, Bristol-Myers Squibb, Prague, Czech Republic, 16 g/day) and chenodeoxycholic acid (CDCA; Chenofalk ${ }^{\circledR}$, Dr. Falk Pharma GmbH, Freiburg, Germany, 1-1.5 g/day dependent on the weight of the subject). One day before each of these examinations, the first blood sample was drawn at 7 AM (-24h) and subjects received food for the whole day to standardize their intake before the study. On the day of examination, the first blood sample was drawn again at $7 \mathrm{AM}(0 \mathrm{~h})$ and the blood samples were then collected in 90-min intervals for $15 \mathrm{~h}$ till $10 \mathrm{PM}$. Again, subjects received food for the whole day and they had to eat at exactly defined intervals (breakfast at 7:15, snack at 9:45, lunch at 12:30, snack at 15:30 and dinner at 17:30). The amount of food was calculated to cover their energy requirements; the diet was relatively low in fat $(25 \%$ of energy intake). If the examination included the drug administration, the drugs were given to subjects on the day before the examination and also on the day of the examination. Questran ${ }^{\circledR}$ was given to subjects in two doses on both days - one with breakfast, the other one with dinner. Due to differences in pharmacokinetics, Chenofalk ${ }^{\circledR}$ treatment was started with dinner on the day preceding the examination and, on the day of examination, it was given to subjects in two doses at the same time as cholestyramine. The order of the examinations was randomized and they were carried out in three-week intervals at a minimum.

The study protocol was approved by the Ethical Committee of the Institute for Clinical and Experimental Medicine and all the participants gave their informed consent.

\section{Biochemistry}

Cholesterol and triglycerides (TG) were measured using enzymatic kits from Roche Diagnostics GmbH, Mannheim, Germany, glucose using kits from PLIVA-Lachema Diagnostika, Brno, Czech Republic, nonesterified fatty acids using kits from Wako Chemicals GmbH, Neuss, Germany, and bile acids using enzymatic kits from Trinity Biotech plc, Bray, Ireland. Insulin was determined using IRMA kits from Immunotech, Prague, Czech Republic. Concentration of $7 \alpha$-hydroxycholest4en-3-one (C4) was determined by HPLC as described by Gälman et al. (2003) with a modification of C4 extraction procedure (Leníček et al. 2008).

\section{Statistics}

The differences between examinations were evaluated using ANOVA for repeated measures and if statistically significant differences were detected, a paired $\mathrm{t}$ test with Bonferroni correction was used to identify those differences. The differences between $-24 \mathrm{~h}$ and $0 \mathrm{~h}$ were evaluated using a paired $t$ test. The relationship between area under curve (AUC) of C4 and AUC of BA and insulin was tested using simple linear regression analysis.

\section{Results}

As expected, C4 concentration as a marker of CYP7A1 activity displayed marked diurnal variation (Fig. 1). The enzyme activity can vary in the order of magnitude within a few hours; the peak was reached around 1 PM in most of our subjects, but, as can be seen, the interindividual variation was rather very high. The one day treatment with cholestyramine resulted in a several fold increase in fasting $\mathrm{C} 4$ concentration on the day of examination and approximately a fivefold increase in the enzyme activity as assessed on the basis of AUC of C4 (Table 1, Fig. 2A). Conversely, the treatment with $\mathrm{CDCA}$ resulted in a decrease of $\mathrm{C} 4$ fasting concentration and $\mathrm{AUC}$ of $\mathrm{C} 4$ to approximately one third throughout 
the day (Table 1, Fig. 2A). The BA concentration varied throughout the day with minor peaks reflecting food intake during lunch and dinner (Fig. 2B). The effects of both treatments on serum BA concentration were not so pronounced - there was a decrease in both fasting BA concentration and AUC BA after cholestyramine and, conversely, approximately a $30 \%$ increase in AUC of BA after CDCA treatment (Table 1, Fig. 2B).

Table 1. The concentration of cholesterol, triglycerides, glucose, nonesterified fatty acids (NEFA), insulin, bile acids (BA), and $7 \alpha$ hydroxycholest-4en-3-one (C4) at 7:00 AM on the day before the study $(-24 \mathrm{~h})$, at 7:00 AM on the day of the study $(0 \mathrm{~h})$ and $15-\mathrm{h}$ area under the curve (AUC) of these variables (AUC [0-15 h]).

\begin{tabular}{|c|c|c|c|c|}
\hline & & $-24 h$ & $\mathbf{O} \mathbf{h}$ & $\begin{array}{c}\text { AUC } \\
{[0-15 \text { h] }}\end{array}$ \\
\hline \multirow{3}{*}{$\begin{array}{l}\text { Cholesterol } \\
\text { (mmol/l) }\end{array}$} & $\mathrm{C}$ & $4.66 \pm 0.94$ & $4.60 \pm 0.96$ & $66.1 \pm 13.5$ \\
\hline & Q & $4.73 \pm 0.76$ & $4.59 \pm 0.86$ & $64.4 \pm 11.5$ \\
\hline & CDCA & $4.65 \pm 0.91$ & $4.63 \pm 0.91$ & $66.8 \pm 12.5$ \\
\hline \multirow{3}{*}{$\begin{array}{l}\text { Triglyceride } \\
(\mathrm{mmol} / \mathrm{l})\end{array}$} & $\mathrm{C}$ & $1.64 \pm 0.74$ & $1.57 \pm 1.03$ & $27.2 \pm 16.4$ \\
\hline & Q & $1.56 \pm 0.95$ & $1.76 \pm 0.86$ & $27.5 \pm 14.9$ \\
\hline & CDCA & $1.62 \pm 0.85$ & $1.61 \pm 0.84$ & $28.3 \pm 12.2$ \\
\hline \multirow{3}{*}{$\begin{array}{l}\text { Glucose } \\
\text { (mmol/l) }\end{array}$} & $\mathrm{C}$ & $5.10 \pm 0.46$ & $5.11 \pm 0.45$ & $80.3 \pm 6.8^{\mathrm{a}}$ \\
\hline & $\mathrm{Q}$ & $5.26 \pm 0.63$ & $5.09 \pm 0.41$ & $71.5 \pm 5.9^{\mathrm{b}}$ \\
\hline & $\mathrm{CDCA}$ & $5.45 \pm 0.96$ & $4.96 \pm 0.59$ & $74.9 \pm 9.2^{\mathrm{a}}$ \\
\hline \multirow{3}{*}{$\begin{array}{l}\text { NEFA } \\
(\mathrm{mmol} / \mathrm{l})\end{array}$} & $\mathrm{C}$ & $0.37 \pm 0.27$ & $0.43 \pm 0.15$ & $3.32 \pm 0.98$ \\
\hline & Q & $0.37 \pm 0.26$ & $0.55 \pm 0.50$ & $3.03 \pm 1.69$ \\
\hline & CDCA & $0.30 \pm 0.16$ & $0.50 \pm 0.32 *$ & $2.31 \pm 1.49$ \\
\hline \multirow{3}{*}{$\begin{array}{l}\text { Insulin } \\
(I U / l)\end{array}$} & $\mathrm{C}$ & $7.8 \pm 6.0$ & $6.3 \pm 3.7$ & $275 \pm 133$ \\
\hline & Q & $8.0 \pm 3.3$ & $9.7 \pm 6.4$ & $220 \pm 124$ \\
\hline & CDCA & $9.8 \pm 9.5$ & $8.0 \pm 5.7$ & $265 \pm 141$ \\
\hline \multirow{3}{*}{$\begin{array}{l}\text { BA } \\
(\mu \mathrm{mol} / \mathrm{l})\end{array}$} & $\mathrm{C}$ & $13.4 \pm 6.1$ & $11.0 \pm 4.5^{\mathrm{a}, \mathrm{b}}$ & $186 \pm 64^{\mathrm{a}}$ \\
\hline & Q & $11.4 \pm 3.8$ & $8.7 \pm 2.9 *^{\mathrm{a}}$ & $133 \pm 37^{b}$ \\
\hline & CDCA & $12.6 \pm 4.7$ & $13.1 \pm 4.0^{\mathrm{b}}$ & $242 \pm 86^{\mathrm{a}}$ \\
\hline \multirow{3}{*}{$\begin{array}{l}C 4 \\
(\mu g / l)\end{array}$} & $\mathrm{C}$ & $20.1 \pm 22.0$ & $25.3 \pm 23.0^{\mathrm{a}}$ & $303 \pm 168^{\mathrm{a}}$ \\
\hline & $\mathrm{Q}$ & $22.7 \pm 17.6$ & $86.9 \pm 49.8^{* * b}$ & $1512 \pm 784^{b}$ \\
\hline & CDCA & $30.2 \pm 24.9$ & $11.8 \pm 13.0 * * \mathrm{c}$ & $119 \pm 70^{\mathrm{c}}$ \\
\hline
\end{tabular}

Data are mean \pm S.D. C - control experiment, Q cholestyramine treatment, CDCA - chenodeoxycholic acid treatment. *,** $\mathrm{p}<0.05, \mathrm{p}<0.01 \ldots-24$ hours vs. 0 hours using paired t-test. $a, b, c \ldots$ the same letters are assigned to the examinations that do not differ when $C, Q$, and CDCA are compared using ANOVA for repeated measures $(p<0.05)$.

The treatment with both drugs had no effect on both fasting value and AUC of cholesterol, TG, and insulin (Table 1, Fig. 1). The fasting concentration of NEFA rose after CDCA treatment but did not differ from
Table 2. Correlation coefficients $r$ of the relationships between AUC of $7 \alpha$-hydroxycholest-4-en-3-one (C4) and AUC of bile acids (BA) and AUC of insulin in subjects in control examination (C), after treatment with cholestyramine (Q) and chenodeoxycholic acid (CDCA).

\begin{tabular}{lcccc}
\hline & n & AUC of BA & AUC of insulin & \\
\hline$C$ & 12 & 0.142 & 0.423 & \\
$Q$ & 12 & 0.113 & 0.727 & $* *$ \\
$C D C A$ & 12 & 0.011 & 0.729 & $* *$ \\
\hline
\end{tabular}

$* * p<0.01$.

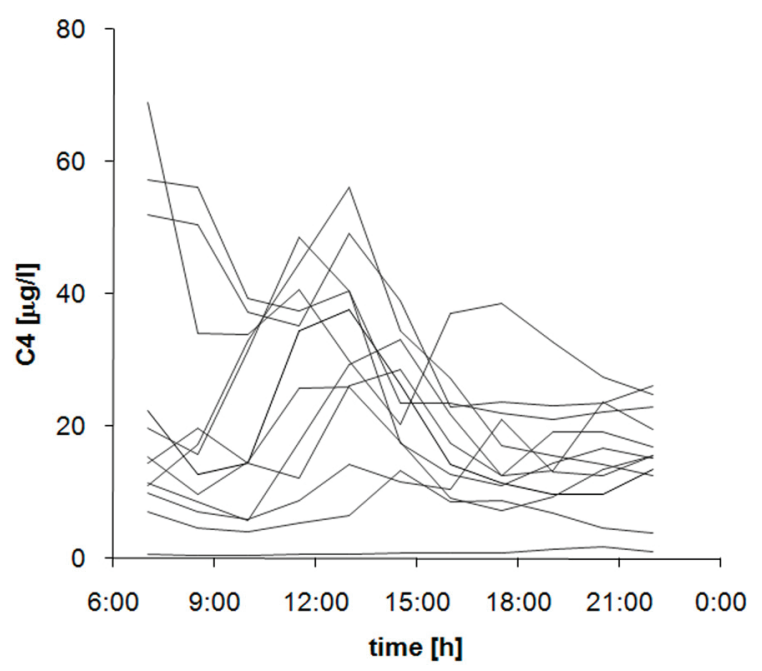

Fig. 1. Diurnal variation of $7 \alpha$-hydroxycholest-4en-3-one (C4) concentration in twelve healthy subjects included into study during control examination (C).

the fasting concentration in cholestyramine or control examinations. However, although the treatment with both drugs had no effect on fasting glucose, the AUC of glucose after cholestyramine was approximately $10 \%$ lower than in control experiment $(\mathrm{p}<0.05)$ (Table 1 , Fig. 2D).

To further analyze the relationship between possible regulatory role that $\mathrm{BA}$ and insulin can exert on BA synthesis, AUC of BA and AUC of insulin (as a measure of their bioavailability) were correlated to AUC of C4. As expected, there was a rather weak but statistically significant negative correlation between AUC $\mathrm{BA}$ and $\mathrm{AUC} \mathrm{C} 4$ when all three examinations in all the subjects were included $(\mathrm{r}=-0.430, \mathrm{p}<0.05)$. However, no such relationship could be found when the treatments were analyzed separately (Table 2). On the contrary, there was a strong positive correlation between AUC of insulin and AUC of $\mathrm{C} 4$ when subjects were treated with cholestyramine and/or CDCA (Table 2). Similar results were obtained when AUC's of BA and insulin obtained 

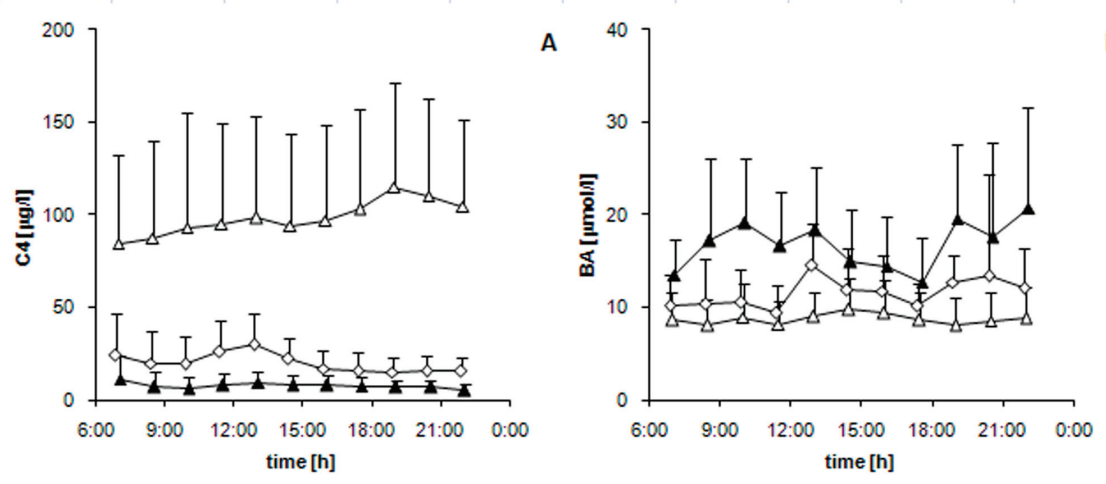

Fig. 2. The diurnal variation of concentration of $7 \alpha$-hydroxycholest-4en3-one (C4), bile acids (BA), insulin, glucose, nonesterified fatty acids (NEFA), and triglyceride in the control experiment (C), after short treatment with cholestyramine (Q) or chenodeoxycholic acid (CDCA). $\diamond-$ control examination (C); $\Delta-$ short treatment with cholestyramine (Q); $\Delta$ - short treatment with chenodeoxycholic acid (CDCA).
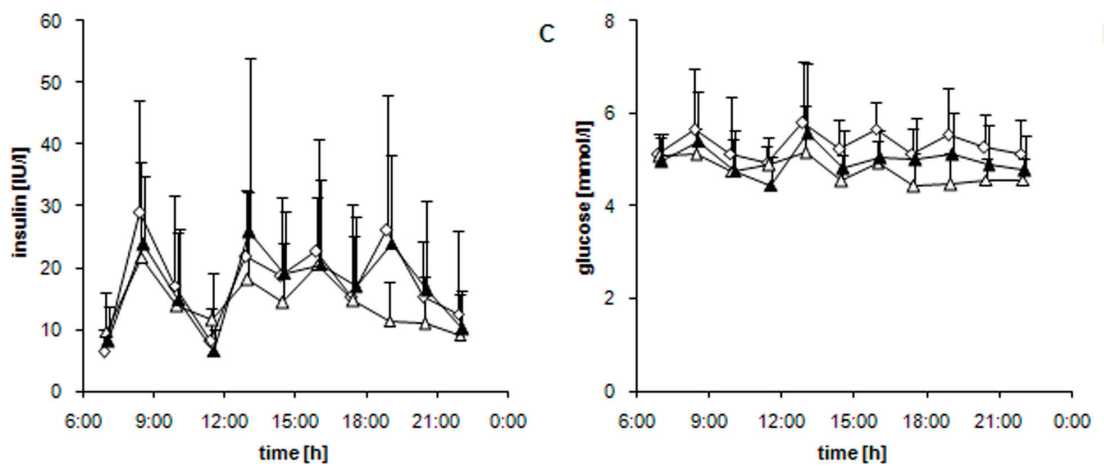

D
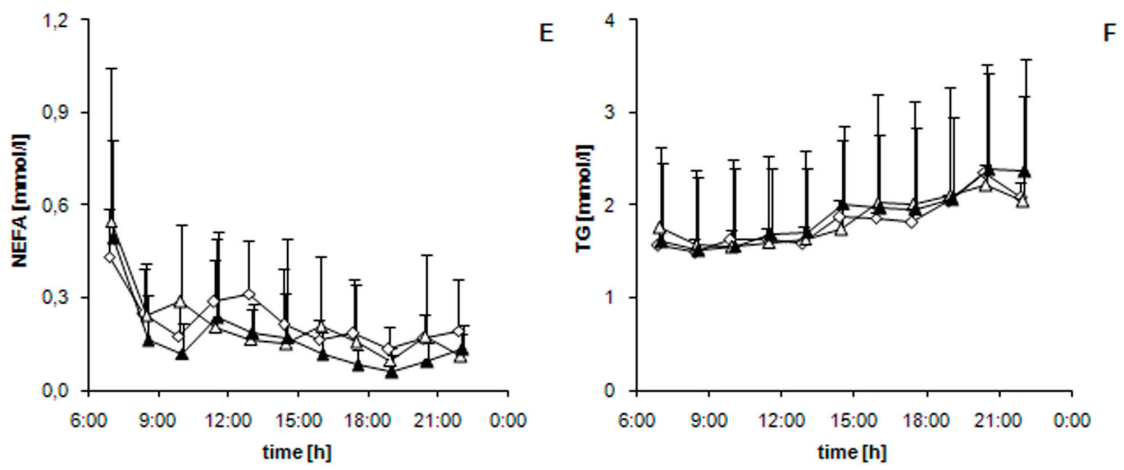

in shorter time intervals (7:00 to $11: 30,7: 00$ to $13: 00$, 7:00 to $14: 30,7: 00$ to $16: 00$ ) were correlated to AUC C4 (data not shown).

\section{Discussion}

In the present study we demonstrated that CYP7A1 activity displays a diurnal variation in healthy men with a peak around 1 PM. The treatment with cholestyramine reduces serum BA concentration and several fold upregulates the CYP7A1 activity and, conversely, the treatment with CDCA increases BA concentration and downregulates its activity. The AUC of $\mathrm{C} 4$ as a measure of bile acid synthetic capacity is positively correlated with AUC of insulin on cholestyramine and CDCA, but unexpectedly not with AUC of BA.
Our findings that $\mathrm{C} 4$ concentration as a marker of CYP7A1 activity displays a peak around a midday confirms the earlier findings from the study of five healthy volunteers (Gälman et al. 2005). However, it should be noted that three out of twelve subjects in our study show maximal C4 concentrations early in the morning (Fig. 1) and we should be cautious to generalize our conclusions about the CYP7A1 activity diurnal variation. Based on our data, some of the subjects may have displayed more than one peak of CYP7A1 activity a day and we cannot even exclude the possibility that in some subjects there may be a peak during the night like in rodents (Noshiro et al. 1990).

If we assume that the short-term administration of cholestyramine upregulates CYP7A1 gene expression to a maximum, then the comparison of areas under the $\mathrm{C} 4$ curves suggest that, on average, the enzyme activity 
throughout the day varies around $20 \%$ of the possible maximum and, thus, the enzyme activity is rather suppressed under physiological conditions (Table 1, Fig. 2A). On the other hand, it is quite surprising that there is no correlation between AUC of BA and that of C4 (Table 2). It may be that the effect of BA on CYP7A1 activity is at its maximum or minimum when the subjects are treated with drugs and this is not related to the concentration of BA anymore. However, the lack of such correlation in the control examination suggests that the effect of other factors on CYP7A1 may prevail. Moreover, given that CYP7A1 activity is rather low for most of the day, it may be difficult to see a further suppressive effect of BA. From the other factors that can override the effect of BA, insulin deserves a special attention. We could indeed demonstrate quite a strong positive correlation between AUC of $\mathrm{C} 4$ as a measure of CYP7A1 activity and AUC of insulin as a measure of its bioavailability at least when both drugs are used to manipulate the transhepatic flux of BA. Such an observation is in agreement with recent findings that insulin in physiological concentrations has a dual effect on CYP7A1 gene expression in human primary hepatocytes (Li et al. 2006). First, it induces a very rapid and pronounced activation of CYP7A1 gene expression likely due to the activation of $\mathrm{p} 38$ MAPK pathway (Xu et al. 2007). Then, after several hours, it inhibits gene expression through the effect of two insulin-regulated transcriptional factors - forkhead box $\mathrm{O} 1$ (FoxO1) and sterol regulatory element binding protein-1c (SREBP-1c) - on transactivation of the CYP7A1 gene (Li et al. 2006).

Therefore, we can hypothesize that insulin could be the first signal to induce CYP7A1 gene expression at the transition from fasting to postprandial phase. Afterwards, the effect of BA return from enterohepatic cycle, FGF19 signaling, and downregulating effect of insulin should prevail and lower the CYP7A1 activity. Although such a hypothesis fits with our data, we are fully aware of the fact that insulin is only one of many factors that are involved in the complex regulation of transition to the postprandial state. It was shown that the circadian variation of CYP7A1 activity is preserved even in fasting subjects that are allowed to drink only water (Gälman et al. 2005) which suggests that the other mechanisms such as signalling through albumin D-element-binding protein (DBP) (Lavery and Schibler 1993) or through FGF19 (Lundasen et al. 2006) may be involved in the regulation of bile acid synthesis during the day.

Our findings that plasma glucose concentration is suppressed after a $24-\mathrm{h}$ treatment with cholestyramine is in line with the observation that a 6-week treatment of diabetic patients with cholestyramine improved glycemia and glycated hemoglobin (Garg and Grundy 1994) and with the findings that bile acids may directly inhibit gluconeogenesis by affecting the transcription of its key regulatory enzyme, phosphoenolpyruvate carboxykinase (PEPCK) (De Fabiani et al. 2003). The mechanism of this effect is not FXR-dependent. Our data suggest for the first time that the effect of low transhepatic flux of bile acid on gluconeogenesis is immediate and likely does not include the extrahepatic effects because the variations of insulin and NEFA concentrations throughout the day were not affected by cholestyramine.

In conclusion, we observed a positive correlation between the area under the curve of insulinemia and that of $7 \alpha$-hydroxycholest-4-en-3-one, the surrogate marker of CYP7A1 activity. Our findings support an idea that insulin may play a role in the regulation of bile acid synthesis in humans.

\section{Conflict of Interest}

There is no conflict of interest.

\section{Acknowledgements}

The authors thank to Ms. Janka Nemčeková, Dana Körberová, Jitka Krupková, Dagmar Šafránková, Jaroslava Štochlová, and Dr. Petr Stávek for their excellent technical help. The work was supported by grant No. NR/8987-3 from the Ministry of Health of the Czech Republic.

\section{References}

AXELSON M, ALY A, SJÖVALL J: Levels of $7 \alpha$-hydroxy-4-cholesten-3-one in plasma reflect rates of bile acid synthesis in man. FEBS Lett 239: 324-328, 1988.

CHIANG JYL: Regulation of bile acid synthesis. Front Biosci 3: d176-d193, 1998. 
DE FABIANI E, MITRO N, GILARDI F, CARUSO D, GALLI G, CRESTANI M: Coordinated control of cholesterol catabolism to bile acids and of gluconeogenesis via a novel mechanism of transcription regulation linked to the fasted-to-fed cycle. J Biol Chem 278: 39124-39132, 2003.

GÄLMAN C, ARVIDSSON I, ANGELIN B, RUDLING M: Monitoring hepatic cholesterol 7 $\alpha$-hydroxylase activity by assay of the stable bile acid intermediate $7 \alpha$-hydroxy-4-cholesten-3-one in peripheral blood. $J$ Lipid Res 44 : 859-866, 2003.

GÄLMAN C, ANGELIN B, RUDLING M: Bile acid synthesis in humans has a rapid diurnal variation that is asynchronous with cholesterol synthesis. Gastroenterology 129: 1445-1453, 2005.

GARG A, GRUNDY SM: Cholestyramine therapy for dyslipidemia in non-insulin-dependent diabetes mellitus: A short-term, double-blind, crossover trial. Ann Intern Med 121: 416-422, 1994.

GILARDI F, MITRO N, GODIO C, SCOTTI E, CARUSO D, CRESTANI M, DE FABIANI E: The pharmacological exploitation of cholesterol $7 \alpha$-hydroxylase, the key enzyme in bile acid synthesis: from binding resins to chromatin remodelling to reduce plasma cholesterol. Pharmacol Ther 116: 449-472, 2007.

INAGAKI T, CHOI M, MOSCHETTA A, PENG L, CUMMINS CL, MCDONALD JG, LUO G, JONES SA, GOODWIN BA, RICHARDSON JA, GERARD RD, REPA JJ, MANGELSDORF DJ, KLIEWER SA: Fibroblast growth factor 15 functions as an enterohepatic signal to regulate bile acid homeostasis. Cell Metab 2: $217-225,2005$.

LAVERY DJ, SCHIBLER U: Circadian transcription of the cholesterol $7 \alpha$ hydroxylase gene may involve the liverenriched bZIP protein DBP. Genes Dev 7: 1871-1884, 1993.

LENÍČEK M, JUKLOVÁ M, ZELENKA J, KOVÁŘ J, LUKÁŠ J, BORTLÍK M, VÍTEK L: Improved HPLC analysis of serum 7 $\alpha$-hydroxycholest-4-en-3-one, a marker of bile acid malabsorption. Clin Chem 54: 1087-1096, 2008.

LI T, KONG X, OWSLEY E, ELLIS E, STROM S, CHIANG JYL: Insulin regulation of cholesterol 7 $\alpha$-hydroxylase expression in human hepatocytes. Roles of forkhead box $\mathrm{O} 1$ and sterol regulatory element-binding protein $1 \mathrm{c}$. J Biol Chem 281: 28745-28754, 2006.

LUNDASEN T, GÄLMAN C, ANGELIN B, RUDLING M: Circulating intestinal fibroblast growth factor 19 has a pronounced diurnal variation and modulates hepatic bile acid synthesis. J Intern Med 260: 530-536, 2006.

MA K, SAHA PK, CHAN L, MOORE DD: Farnesoid X receptor is essential for normal glucose homeostasis. $J$ Clin Invest 116: 1102-1109, 2006.

NOSHIRO M, NISHIMOTO M, OKUDA K: Rat liver cholesterol $7 \alpha$-hydroxylase. Pretranslational regulation for circadian rhythm. J Biol Chem 265: 10036-10041, 1990.

RAMIREZ MI, KARAOGLU D, HARO D, BARILLAS C, BASHIRZADEH R, GIL G: Cholesterol and bile acids regulate cholesterol $7 \alpha$-hydroxylase expression at the transcriptional level in culture and transgenic mice. Mol Cell Biol 14: 2809-2821, 1994.

XU Z, TAVARES-SANCHEZ OL, LI Q, FERNANDO J, RODRIGUEZ CM, STUDER EJ, PANDAK WM, HYLEMON PB, GIL G: Activation of bile acid synthesis by the p38 mitogen-activated protein kinase (MAPK). Hepatocyte nuclear factor- $4 \alpha$ phosphorylation by the p38 MAPK is required for cholesterol $7 \alpha-$ hydroxylase expression. J Biol Chem 282: 24607-24614, 2007. 